\title{
Room temperature quantum spin Hall states in two-dimensional crystals composed of pentagonal rings and their quantum wells
}

\author{
Yandong $\mathrm{Ma}^{1}$, Liangzhi $\mathrm{Kou}^{2}$, Xiao $\mathrm{Li}^{3}$, Ying $\mathrm{Dai}^{4}$ and Thomas Heine ${ }^{1,5}$ \\ Quantum spin Hall (QSH) insulators are a peculiar phase of matter exhibiting excellent quantum transport properties with \\ potential applications in lower-power-consuming electronic devices. Currently, among all predicted or synthesized QSH \\ insulators, square and hexagonal atomic rings are the dominant structural motifs, and QSH insulators composed of pentagonal \\ rings have not yet been reported. Here, based on first-principles calculations, we predict a family of large-gap QSH insulators \\ in $\mathrm{SnX}_{2}\left(\mathrm{X}=\mathrm{S}\right.$, Se, or Te) two-dimensional (2D) crystals by the direct calculation of $\mathrm{Z}_{2}$ topological invariants and edge states. \\ Remarkably, in contrast to all known QSH insulators, the QSH insulators predicted here are composed entirely of pentagonal \\ rings. Moreover, these systems can produce sizeable nontrivial gaps ranging from 121 to $224 \mathrm{meV}$, which is sufficiently large \\ for practical applications at room temperature. Additionally, we propose a quantum well by sandwiching an $\mathrm{SnTe}_{2} 2 \mathrm{D}$ crystal \\ between two $\mathrm{BiOBiS}_{2}$ sheets and reveal that the considered $2 \mathrm{D}$ crystal remains topologically nontrivial with a sizeable gap. This \\ finding demonstrates the robustness of its band topology against the effect of the substrate and provides a viable method for \\ further experimental studies.
}

NPG Asia Materials (2016) 8, e264; doi:10.1038/am.2016.51; published online 15 April 2016

\section{INTRODUCTION}

Topological insulators (TIs) have sparked extensive research activities in recent years because of their rich physics and promising applications in quantum devices and spintronics. ${ }^{1,2}$ The resultant Dirac surface states in three-dimensional (3D) TIs as well as the helical edge states in two-dimensional (2D) TIs are spin locked because of protection by time-reversal symmetry; thus, they are robust against perturbations. Particularly in 2D TIs, also known as quantum spin Hall (QSH) insulators, ${ }^{3,4}$ all the low-energy scatterings of the edge states caused by the nonmagnetic defects are completely forbidden because the edge electrons can only propagate along two directions with opposite spins, which makes 2D TIs more suitable for lowpower-consuming applications than 3D TIs. Unfortunately, the material realization of $2 \mathrm{D}$ TIs is challenging: while $3 \mathrm{D}$ TIs have been discovered in many materials, such as the $\mathrm{Bi}_{2} \mathrm{Se}_{3}$ family, ${ }^{5,6}$ the experimental realization of $2 \mathrm{D}$ TIs is thus far limited to the $\mathrm{HgTe} /$ $\mathrm{CdTe}^{7}$ and InAs/GaSb ${ }^{8}$ quantum wells. Moreover, the QSH effect in these two quantum wells can occur only at ultralow temperature $(<10 \mathrm{~K})$ because of their extremely small bulk band gaps, and this limitation greatly obstructs their possible applications. The search for new 2D TIs with large band gaps that could support room temperature applications has thus become critically important.
Considerable effort has been devoted thus far to search for new 2D materials or new schemes to realize 2D TIs, and a variety of large-gap 2D TIs have been proposed theoretically. These large-gap 2D TIs include silicene, ${ }^{9} \mathrm{Bi}\left(\begin{array}{lll}1 & 1 & 1\end{array}\right)$ bilayers, ${ }^{10}$ III-Bi bilayers, ${ }^{11} \mathrm{BiF} 2 \mathrm{D}$ crystals, ${ }^{12} \mathrm{Bi}_{4} \mathrm{Br}_{4}$ single layers, ${ }^{13}$ chemically modified $\mathrm{Ge} / \mathrm{Sn}^{14-16}$ and $\mathrm{Bi} / \mathrm{Sb}^{17,18}$ honeycomb lattices, $\mathrm{ZrTe}_{5} / \mathrm{HfTe}_{5}{ }^{19}$ and $2 \mathrm{D}$ transition metal dichalcogenides. ${ }^{20-23}$ In terms of geometrical motifs, square, hexagonal and pentagonal rings are considered to be the three basic building blocks of 2D materials. However, among all predicted or synthesized 2D TIs, ${ }^{9-23}$ square and hexagonal rings are almost the only building blocks that have been found. Such a limitation mostly arises from the fact that the presence of a gapless band structure in these 2D materials makes them promising for harboring QSH states. Therefore, the common expectation is that the QSH effect cannot survive in pentagonal ring-based 2D crystals. To the best of our knowledge, extended 2D TIs composed exclusively of pentagonal rings have never been reported at all, although recent efforts have been conducted to search for such pentagonal ring-based $2 \mathrm{D}$ materials. ${ }^{24,25}$

The purpose of this work is to explore novel 2D topological phases, especially at room temperature, in the supposedly ordinary pentagonal ring-based $2 \mathrm{D}$ crystals. The practical means of doing so will not only significantly enrich our fundamental understanding of the topological phenomena but also greatly broaden the possibilities for realistic

\footnotetext{
${ }^{1}$ Department of Physics and Earth Sciences, Jacobs University Bremen, Bremen, Germany; ${ }^{2}$ School of Chemistry, Physics and Mechanical Engineering Faculty, Queensland University of Technology, Brisbane, QLD, Australia; ${ }^{3}$ Department of Physics, University of Texas at Austin, Austin, TX, USA; ${ }^{4}$ School of Physics, Shandong University, Jinan, People's Republic of China and ${ }^{5}$ Wilhelm-Ostwald-Institut für Physikalische und Theoretische Chemie, Universität Leipzig, Leipzig, Germany

Correspondence: Dr Y Ma or Professor T Heine, Department of Physics and Earth Sciences, Jacobs University Bremen, Campus Ring 1, 28759 Bremen, Germany.

E-mail: myd1987@gmail.com or thomas.heine@uni-leipzig.de

Received 21 October 2015; revised 25 January 2016; accepted 21 February 2016
} 
applications of the QSH effect. After extensive research, we show that 2D TIs, consisting entirely of pentagonal rings, can indeed exist, namely in the family of $\mathrm{SnX}_{2} 2 \mathrm{D}$ crystals, in which $\mathrm{X}=\mathrm{S}$, Se or Te. These $\mathrm{SnX}_{2}$ systems are identified to be room-temperature 2D TIs with sizeable nontrivial band gaps ranging from 121 to $224 \mathrm{meV}$, and they form robust QSH systems. These new QSH insulators present single Dirac cone edge states crossing the bulk band gap, which is ideal for dissipationless transport. More notably, by sandwiching the $\mathrm{SnTe}_{2}$ $2 \mathrm{D}$ crystal between two $\mathrm{BiOBiS}_{2}$ sheets, we propose a promising quantum well configuration in which the considered 2D crystal remains topologically nontrivial with a sizeable band gap, thus inducting the robustness of its band topology against the effect of the substrate.

\section{METHODS}

Density functional theory (DFT) calculations are performed using the plane wave basis Vienna $a b$ initio simulation package. ${ }^{26,27}$ The exchange-correlation potential is described by the Perdew-Burke-Ernzerhof ${ }^{28}$ version of the generalized gradient approximation. ${ }^{29}$ The plane-wave cutoff is set to $500 \mathrm{eV}$, and the convergence threshold for energy is set to $10^{-6} \mathrm{eV}$. Monkhorst-Pack $k$-point meshes ${ }^{30}$ of $9 \times 9 \times 1$ and $13 \times 13 \times 1$ are used for geometry optimization and self-consistent electronic structure calculations, respectively. The $2 \mathrm{D}$ crystals are modeled by the periodic slab approach with a vacuum layer of more than $18 \AA$ to ensure decoupling between periodic images. All structures, including the lattice parameters and the internal ion coordinates, are fully optimized with a residual force tolerance of $0.01 \mathrm{eV} \AA^{-1}$. The spin-orbit coupling (SOC) is included in the self-consistent electronic structure calculations. For the quantum well structure, the van der Waals interaction is included by using the density functional theory-D2 approach. ${ }^{31}$

\section{RESULTS}

The schematic crystal structures of $\mathrm{SnX}_{2} 2 \mathrm{D}$ crystals are shown in Figures $1 \mathrm{a}$ and $\mathrm{b}$. We refer to these two configurations as $\alpha$ - and $\beta$-SnX $X_{2}$, respectively. $\beta$-SnX $X_{2}$ is more stable than $\alpha-S n X_{2}$ by $0.7,22.8$ and $25.8 \mathrm{meV}$ per atom in energy for $\mathrm{X}=\mathrm{S}$, Se and Te, respectively. Supplementary Table $\mathrm{S} 1$ gives the corresponding structural details. From the top view, we can see that both $\alpha$ - and $\beta-\mathrm{SnX}_{2} 2 \mathrm{D}$ crystals resemble the structure of experimentally identified layered silver azide $^{32}$ and are composed entirely of the pentagonal rings; they present an amazing pattern that is well known as Cairo pentagonal tiling. ${ }^{33}$ The crystal structure of $\beta-\mathrm{SnX}_{2}$ displays $\mathrm{P}-42{ }_{1} \mathrm{~m}$ symmetry (point group $\mathrm{D}_{2 \mathrm{~d}}$ ) and a square lattice that contains two $\mathrm{Sn}$ and four $\mathrm{X}$ atoms in one unit cell. Its unit cell can be considered a triple-layer structure with an Sn layer sandwiched between two X layers, as shown in Figure 1b. $\alpha-\mathrm{SnX}_{2} 2 \mathrm{D}$ crystals also present a square lattice, but the symmetry is $\mathrm{P} 4 / \mathrm{mbm}$ (point group $\mathrm{D}_{4 \mathrm{H}}$ ). This indicates that $\alpha-\mathrm{SnX}_{2}$ $2 \mathrm{D}$ crystals have inversion symmetry, whereas the inversion symmetry does not hold for the $\beta-S_{n} X_{2} 2 \mathrm{D}$ crystals. Figure 1c shows the first Brillouin zone of $\mathrm{SnX}_{2} 2 \mathrm{D}$ crystals, which is also square with four $X$ points on the side centers and four $M$ points on the corners. These systems predicted here are different from the known $\mathrm{SnX}_{2} 2 \mathrm{D}$ crystals (labeled as $\mathrm{T}-\mathrm{SnX} \mathrm{X}_{2}$ ). The $\mathrm{T}-\mathrm{SnX} \mathrm{X}_{2} 2 \mathrm{D}$ crystals display the $\mathrm{CdI}_{2}$-type structure with a hexagonal lattice instead of a square lattice. The symmetry space group of $\mathrm{T}-\mathrm{SnX} \mathrm{X}_{2} 2 \mathrm{D}$ crystals is $\mathrm{P}-3 \mathrm{~m} 1$ (point group $\mathrm{D}_{3 \mathrm{D}}$ ), and it contains one $\mathrm{Sn}$ and two $\mathrm{X}$ atoms in the unit cell. For more details, please see Supplementary Figure S1 and the corresponding discussion.

The electronic band structures of $\mathrm{SnX}_{2} 2 \mathrm{D}$ crystals without and with the inclusion of SOC are shown in Figure 2. Evidently, the valence and conduction bands away from the $\Gamma$ point are well separated. Thus, we only need to focus on the bands around the $\Gamma$ point. By projecting the bands onto different atomic orbitals, we find that the bands around the Fermi level are mainly contributed by $p_{z}{ }^{-}$, degenerate $p_{z}{ }^{+}, p_{x, y}{ }^{+}$ and degenerate $p_{x}, y^{-}$orbitals of the $\mathrm{Sn} / \mathrm{X}$ atoms. Especially for $\alpha-\mathrm{SnSe}_{2} / \mathrm{SnTe}_{2} 2 \mathrm{D}$ crystals at the $\Gamma$ point, when excluding SOC, the Fermi level separates the $\left(p_{x, y}{ }^{+}\right.$, degenerate $\left.p_{x, y}{ }^{-}\right)$orbitals from the $\left(p_{z}{ }^{-}\right.$, degenerate $\left.p_{z}{ }^{+}\right)$orbitals, with the $\left(p_{x}, y^{+}\right.$, degenerate $\left.p_{x, y^{-}}\right)$ orbitals located above the $\left(p_{z}{ }^{-}\right.$, degenerate $\left.p_{z}{ }^{+}\right)$orbitals. Furthermore, for these two 2D crystals without including SOC, one energy band with the $p_{x, y}{ }^{-}$orbital character overlaps with another energy band with the $p_{z}{ }^{-}$orbital character around the Fermi level, as shown in Figures $2 b$ and $c$. This band overlap leads to the appearance of two band crossings located in very close proximity to the Fermi level near the $\Gamma$ point for $\alpha-\mathrm{SnSe}_{2} / \mathrm{SnTe}_{2} 2 \mathrm{D}$ crystals. Such band crossings can be understood as a result of the symmetry incompatibility of these two bands. Alternately, compared with $\alpha-\mathrm{SnSe}_{2} / \mathrm{SnTe}_{2} 2 \mathrm{D}$ crystals, the bands around the Fermi level are markedly modified in $\beta-\mathrm{SnSe}_{2} / \mathrm{SnTe}_{2}$ $2 \mathrm{D}$ crystals. For $\beta-\mathrm{SnSe}_{2} / \mathrm{SnTe}_{2} 2 \mathrm{D}$ crystals, the energy band with the $p_{z}{ }^{-}$orbital character is located far above the Fermi level, which leaves the degenerate $p_{x, y}{ }^{-}$orbitals on the Fermi level. As a result, without including SOC, $\beta$-SnSe $2 / \mathrm{SnTe}_{2} 2 \mathrm{D}$ crystals are gapless, and the valence band maximum and conduction band minimum degenerate at the Fermi level at the $\Gamma$ point.

After including SOC, for $\alpha-\mathrm{SnX}_{2} 2 \mathrm{D}$ crystals, the band gap opens at the band crossing; see Figures $2 \mathrm{a}-\mathrm{c}$. The systems have indirect band gaps of 130, 224 and $121 \mathrm{meV}$ for $\mathrm{X}=\mathrm{S}$, Se and Te, respectively (see Figure 1d). To understand the role of SOC on the band structures, we show the schematic diagram of the band evolution at the $\Gamma$ point of $\alpha-\mathrm{SnSe}_{2}$ and $\alpha-\mathrm{SnTe}_{2} 2 \mathrm{D}$ crystals under SOC in Figure 1e. $\lambda$, $\xi$ and $\delta$ in Figure 1e denote the band gap without SOC, the band splitting between degenerate $p_{x}, y^{-}$orbitals, and the band gap with SOC, respectively. We focus on the $p_{z}^{-}$, degenerate $p_{z}^{+}, p_{x}, y^{+}$and degenerate $p_{x, y^{-}}$orbitals around the Fermi level and neglect other atomic orbitals; see Figure 1e. Here, the superscripts ' + ' and '-' represents the bonding and antibonding states, respectively. For $\alpha-\mathrm{SnSe}_{2} / \mathrm{SnTe}_{2} 2 \mathrm{D}$ crystals, without including SOC, one $p_{z}{ }^{-}$orbital lies below the degenerate $p_{x, y}{ }^{-}$orbitals, and the Fermi level stays between them; see Figure 1e. When switching on SOC, the degenerate $p_{x, y}{ }^{-}$orbitals split, and the order of one $p_{x, y}{ }^{-}$orbital and the $p_{z}{ }^{-}$ orbital is exchanged. According to the band overlap features shown in Figures $2 \mathrm{~b}$ and $\mathrm{c}$, it seems that such nontrivial band inversion before including SOC is induced by the inversion between the $p_{z}{ }^{-}$and $p_{x, y}{ }^{-}$ orbitals near the Fermi level at the $\Gamma$ point; however, this is not that case. We know that a band inversion that can affect the band topology, namely a nontrivial band inversion, refers to the inversion between bands with different parities. Otherwise, if a band inversion occurs between bands with the same parity, then it is a trivial band inversion; therefore, it could not affect the band topology. Our parity analysis shows that the $p_{z}{ }^{-}$and $p_{x, y}{ }^{-}$orbitals all display '-' parity, which indicates that a nontrivial band inversion can never be induced by the inversion between these orbitals. In other words, here, the band overlap features near the Fermi level, as shown in Figures $2 b$ and $c$, have nothing to do with the intrinsic nontrivial band inversion. Actually, the nontrivial band order before including SOC in these systems stems from the band inversion between the $\left(p_{z}{ }^{-}\right.$, degenerate $\left.p_{z}^{+}\right)$and $\left(p_{x, y^{+}}\right.$, degenerate $\left.p_{x, y^{-}}\right)$orbitals, which is caused by the crystal field. To elucidate the underlying band inversion mechanism explicitly, we display the orbital evolution at the $\Gamma$ point under the crystal field effect in Figure 1e (namely, from (I) to (II)). The crystal field effect is estimated by comparing the orbital orders with those of the same crystal that is strained to $170 \%$, as suggested by Zhou et al. ${ }^{34}$ and Huang et al. ${ }^{35}$ Without including the crystal field effect (see (I) of Figure 1e), the $\left(p_{z}{ }^{-}\right.$, degenerate $\left.p_{z}{ }^{+}\right)$orbitals are located above the 
a

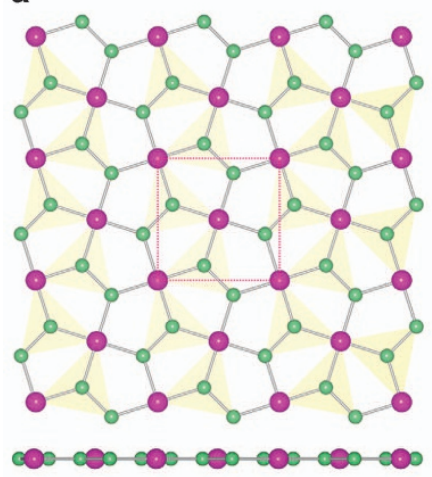

b

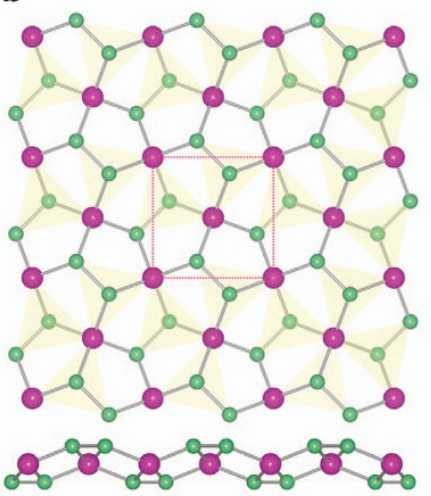

d

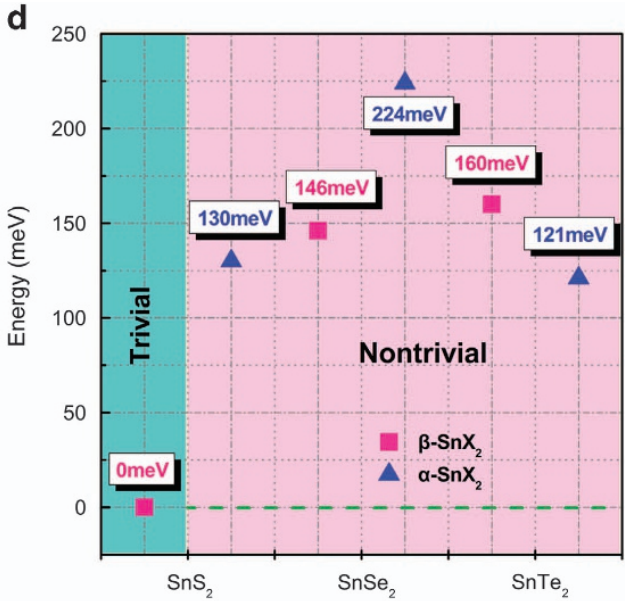

C

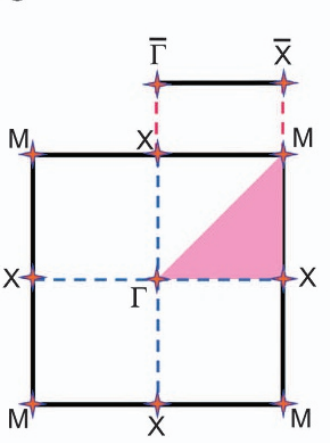

e

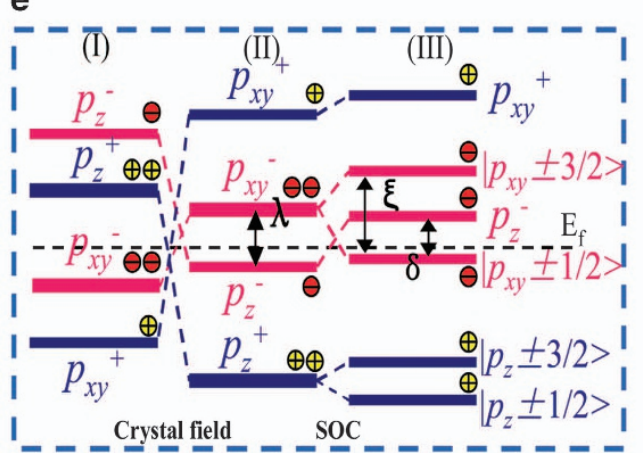

f

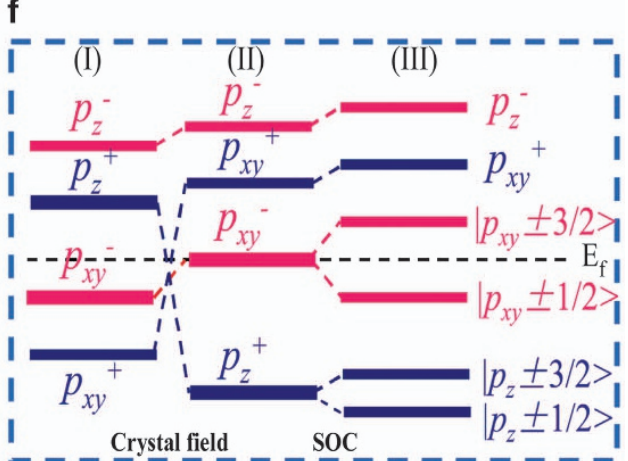

Figure 1 Top and side views of the schematic crystal structures of (a) $\alpha$ - and (b) $\beta$-SnX $X_{2}$ two-dimensional (2D) crystals displaying the unit cell with the red dashed lines. Pink and cyan balls denote $\mathrm{Sn}$ and $\mathrm{X}$ atoms, respectively. (c) $2 \mathrm{D}$ and projected one-dimensional (1D) Brillouin zones of $\mathrm{SnX} 2 \mathrm{2D}$ crystals with high-symmetry points labeled. (d) The nontrivial band gaps of $\mathrm{SnX}_{2} 2 \mathrm{D}$ crystals. (e and f) Schematic diagrams of the evolution of energy levels at the $\Gamma$ point for $(\alpha, \beta)-\mathrm{SnSe}_{2} / \mathrm{SnTe}_{2} 2 \mathrm{D}$ crystals under the crystal field effect and spin-orbit coupling (SOC) in sequence. Here, the superscripts + and - represent the bonding and antibonding states, respectively, whereas the + and - in the circles denote the parities. $\lambda, \xi$ and $\delta$ in (e) denote the band gap without SOC, the band splitting between degenerate $p_{x, y^{-}}$orbitals and the band gap with $\mathrm{SOC}$, respectively.

Fermi level, whereas the $\left(p_{x, y^{+}}\right.$, degenerate $\left.p_{x, y^{-}}\right)$orbitals are located below the Fermi level. At this moment, the band topologies of $\alpha-\mathrm{SnSe}_{2} / \mathrm{SnTe}_{2}$ are trivial. Under the crystal field effect (see (II) of Figure 1e), a band inversion occurs between the ( $p_{z}{ }^{-}$orbital with ' - ' parity, degenerate $p_{z}{ }^{+}$orbitals with ' + ' parity) and $\left(p_{x, y}{ }^{+}\right.$orbital with '+' parity, degenerate $p_{x, y}{ }^{-}$orbitals with '-' parity), indicating a topological phase transition from a trivial insulator to a nontrivial insulator. Obviously, the subsequent SOC-induced band exchange between the $p_{z}{ }^{-}$and $p_{x, y}{ }^{-}$orbitals does not contribute to the band inversion because they both display '-' parity. This is different from the situation in the previously reported systems, such as $\mathrm{Bi}_{2} \mathrm{Te}_{3},{ }^{36}$ where the SOC-induced band exchange usually changes the band topology.

To understand the difference in band structures between $\alpha-\mathrm{SnS}_{2}$ and $\alpha-\mathrm{SnSe}_{2} / \mathrm{SnTe}_{2} 2 \mathrm{D}$ crystals, we further display the orbital evolution at the $\Gamma$ point for $\alpha-S_{2} S_{2} 2 \mathrm{D}$ crystal in Supplementary Figure S2. As shown in Supplementary Figure S2, similar to $\alpha-\mathrm{SnSe}_{2} / \mathrm{SnTe}_{2} 2 \mathrm{D}$ crystals, the $\left(p_{z}{ }^{-}\right.$, degenerate $\left.p_{z}{ }^{+}\right)$orbitals also lie above the $\left(p_{x, y}{ }^{+}\right.$, degenerate $p_{x, y}{ }^{-}$) orbitals before including the crystal field effect, and then a band inversion occurs between the $\left(p_{z}{ }^{-}\right.$, degenerate $\left.p_{z}{ }^{+}\right)$and $\left(p_{x, y^{+}}{ }^{+}\right.$, degenerate $\left.p_{x, y}{ }^{-}\right)$orbitals after including the crystal field effect, thus driving the $\alpha-\mathrm{SnS}_{2} 2 \mathrm{D}$ crystal into a topological phase. Here, the SOC also does not contribute to the band inversion. It should be noted that the crystal field effect shifts the $\left(p_{z}{ }^{-}\right.$, degenerate $\left.p_{z}{ }^{+}\right)$ orbitals of the $\alpha-\mathrm{SnS}_{2} 2 \mathrm{D}$ crystal far above the Fermi level with respect to the case of $\alpha-\mathrm{SnSe}_{2} / \mathrm{SnTe}_{2}$, as shown in Supplementary Figure S2. Therefore, the subsequent SOC-induced band exchange between the $p_{z}{ }^{-}$and $p_{x, y}{ }^{-}$orbitals observed in $\alpha-\mathrm{SnSe}_{2} / \mathrm{SnTe}_{2}$ does not occur in the $\alpha-\mathrm{SnS}_{2} 2 \mathrm{D}$ crystal (see Figure 1e and Supplementary Figure S2).

For $\beta-\mathrm{SnSe}_{2}$ and $\beta-\mathrm{SnTe}_{2} 2 \mathrm{D}$ crystals, SOC also lifts the degeneracy of the bands at the Fermi level, and the conduction band shifts upwards while the valence band shifts downwards, which forms a band gap of 146 and $160 \mathrm{meV}$, respectively. The corresponding schematic diagram of the band evolution at the $\Gamma$ point under the crystal field effect and SOC in sequence is plotted in Figure 1f. Before including the crystal field effect (see (I) of Figure 1f), the $\left(p_{z}{ }^{-}\right.$, degenerate $\left.p_{z}{ }^{+}\right)$and $\left(p_{x, y^{+}}\right.$, degenerate $\left.p_{x,} y^{-}\right)$orbitals are located above and below the Fermi level, respectively. After including the crystal field effect (see (II) of Figure 1f), a band inversion occurs between the degenerate $p_{z}{ }^{+}$and $\left(p_{x, y^{+}}\right.$, degenerate $\left.p_{x, y^{-}}\right)$orbitals, indicting a topological phase transition from a normal insulator to a TI. This leaves the Fermi level right on the degenerate $p_{x, y}{ }^{-}$orbitals; subsequently, after turning on SOC, the degeneracy of the two $p_{x, y}{ }^{-}$ orbitals is lifted; see the SOC part of Figure 1f. Therefore, $\beta-\mathrm{SnSe}_{2} /$ $\mathrm{SnTe}_{2} 2 \mathrm{D}$ crystals also display a nontrivial band order before including SOC, which also arises from the crystal field effect. In these systems, the SOC-induced band exchange also does not contribute to the band inversion, and the only effect of SOC is to create energy gaps at the 

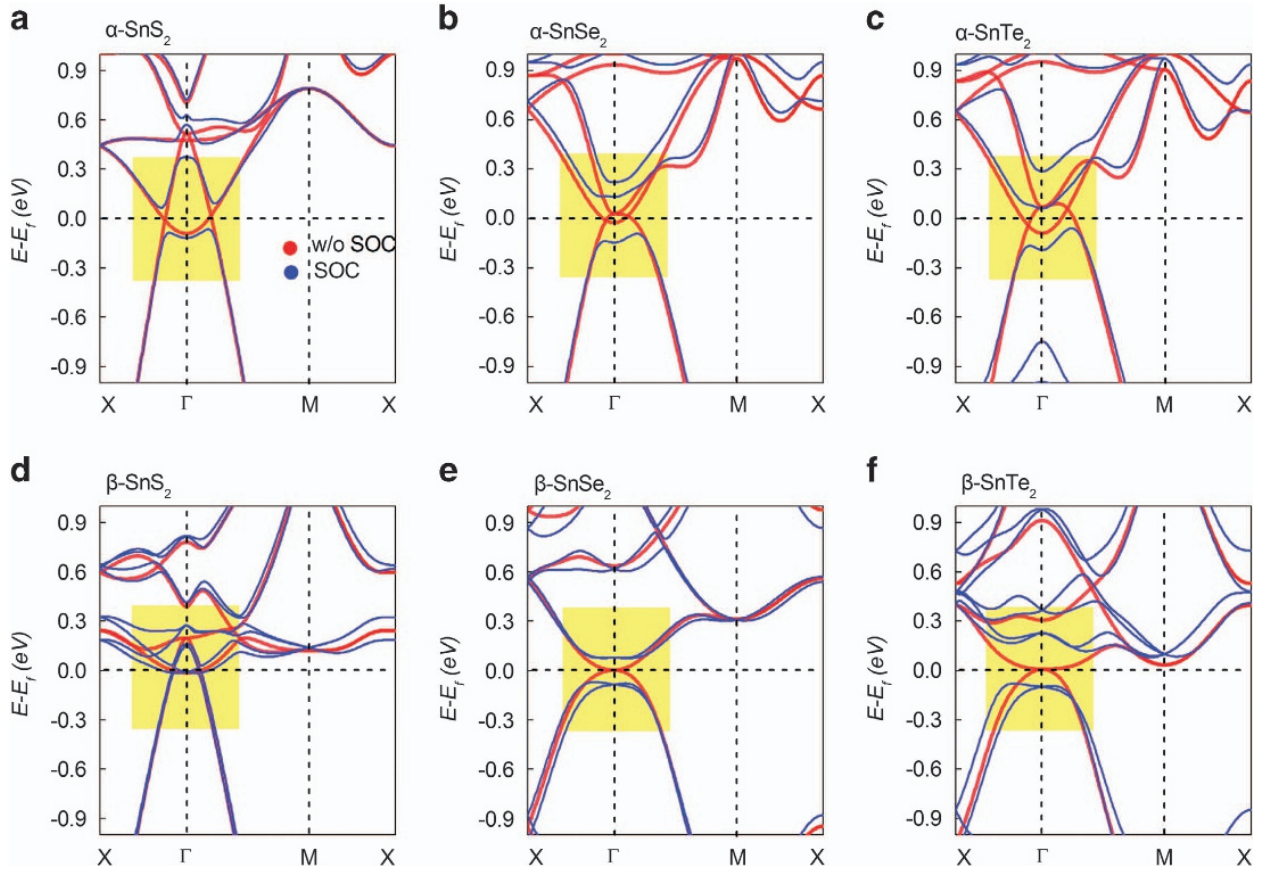

Figure 2 Electronic band structures of (a) $\alpha-\mathrm{SnS}_{2}$, (b) $\alpha-\mathrm{SnSe}_{2}$, (c) $\alpha$-SnTe 2 , (d) $\beta-\mathrm{SnS}_{2}$, (e) $\beta$-SnSe $\mathrm{S}_{2}$ and (f) $\beta$-SnTe 2 two-dimensional (2D) crystals without and with spin-orbit coupling (SOC). Fermi levels are set to zero.

touching points. By comparing Figures $1 \mathrm{e}$ and $\mathrm{f}$, we can see that, without including the crystal field, the orbital order of the $\beta-\mathrm{SnSe}_{2} /$ $\mathrm{SnTe}_{2} 2 \mathrm{D}$ crystals is similar to that of $\alpha-\mathrm{SnSe}_{2} / \mathrm{SnTe}_{2}$, that is, the $\left(p_{z}{ }^{-}\right.$, degenerate $\left.p_{z}^{+}\right)$orbitals are located above the $\left(p_{x, y}{ }^{+}\right.$, degenerate $\left.p_{x, y^{-}}\right)$ orbitals. This is due to the similar elemental composition of $\alpha$ - and $\beta$-SnSe $2 / \mathrm{SnTe}_{2} 2 \mathrm{D}$ crystals. However, as shown in Figures la and b, the geometric structures of the $\alpha$ and $\beta$ phases are very different, which indicates that the crystal field effects in the $\alpha$ - and $\beta-\mathrm{SnSe}_{2} / \mathrm{SnTe}_{2} 2 \mathrm{D}$ crystals are very different. Thus, after including the crystal field effect, a band inversion occurs between the degenerate $p_{z}^{+}$and $\left(p_{x}, y^{+}\right.$, degenerate $p_{x, y}{ }^{-}$) orbitals for $\beta$-SnSe $2 / \mathrm{SnTe}_{2} 2 \mathrm{D}$ crystals, whereas the $\left(p_{z}{ }^{-}\right.$, degenerate $\left.p_{z}{ }^{+}\right)$and $\left(p_{x, y^{+}}\right.$, degenerate $\left.p_{x, y^{-}}\right)$orbitals are inverted for the $\alpha$ phase (see Figures 1e and $\mathrm{f}$ ). As a result, in contrast to the $\alpha$ phase in which the $p_{z}{ }^{-}$orbital shifts below the degenerate $p_{x, y}{ }^{-}$ orbitals, the $p_{z}{ }^{-}$orbital of $\beta-\mathrm{SnSe}_{2} / \mathrm{SnTe}_{2} 2 \mathrm{D}$ crystals remains above the degenerate $p_{x, y^{-}}$orbitals after including the crystal field effect. Moreover, by comparing Figures $2 \mathrm{~d}-\mathrm{f}$, we notice that SOC is responsible for a significant spin splitting of both the valence and conduction bands of $\beta$ $\mathrm{SnX}_{2} 2 \mathrm{D}$ crystals around the Fermi level. The origin lies in the break of inversion symmetry in $\beta-\mathrm{SnX}_{2} 2 \mathrm{D}$ crystals, which induces the local dipole field and thus leads to the spin splitting. Alternately, because $\alpha$ $\mathrm{SnX}_{2} 2 \mathrm{D}$ crystals possess inversion symmetry, bands in Figures $2 \mathrm{a}-\mathrm{c}$ are thus spin degenerate in the presence of SOC. For the $\beta-\mathrm{SnS}_{2} 2 \mathrm{D}$ crystal, the scenario of the band evolution under SOC is very special. This scenario is different from the other five systems because after including SOC, the band gap does not open, and the entire system is metallic. Therefore, for the remainder of our discussion, we will focus on the $\alpha$ $\mathrm{SnX}_{2}, \beta-\mathrm{SnSe}_{2}$ and $\beta-\mathrm{SnTe}_{2} 2 \mathrm{D}$ crystals.

To identify the topological nature of the insulating phases in these systems, we investigate the $Z_{2}$ topological invariants within the density functional theory frame. $Z_{2}=1$ characterizes a topologically nontrivial phase, whereas $Z_{2}=0$ corresponds to a topologically trivial phase. For $\alpha-S n X_{2} 2 D$ crystals, the existence of inversion symmetry simplifies the calculations of $\mathrm{Z}_{2}$ topological invariants. According to the method developed by $\mathrm{Fu}$ and Kane, ${ }^{37}$ topological invariants can be easily obtained by evaluating the parity eigenvalues of each pair of Kramer's degenerate occupied energy bands at the four time-reversal-invariant momenta points (one $\Gamma$, one $M$ and two $X$ ) of the Brillouin zone. Owing to the square symmetry, the parities at the two $X$ points are identical, $\delta(X)^{2} \equiv 1$, which has no effect on the band topology; thus, the topological invariants can be reduced to $(-1)^{\nu}=\delta(\Gamma) \delta(M)$. In $\alpha-\mathrm{SnX}_{2}$ 2D crystals, the product of the parity eigenvalues at the $\Gamma$ point is -1 , whereas it is +1 at the $M$ point; thus, we conclude that $\alpha-S n X_{2} 2 \mathrm{D}$ crystals are nontrivial $2 \mathrm{D}$ TIs with $\mathrm{Z}_{2}=1$. For more details, see Supplementary Table S2. For $\beta-\mathrm{SnSe}_{2}$ and $\beta-\mathrm{SnTe}_{2} 2 \mathrm{D}$ crystals, the inversion symmetry is broken, and the $Z_{2}$ topological invariants cannot be determined by the method mentioned above. Instead, we use the method in Fukui and Hatsugai ${ }^{38}$ to directly perform the lattice computation of the $\mathrm{Z}_{2}$ topological invariants in terms of the $n$-field configuration. The resultant $Z_{2}=1$ verifies that $\beta-\mathrm{SnSe}_{2}$ and $\beta-\mathrm{SnTe}_{2}$ $2 \mathrm{D}$ crystals are also $2 \mathrm{D}$ TIs. The room-temperature QSH effect can be readily realized in all these systems if one considers their nontrivial band gaps. We emphasize that these results go beyond all predicted or synthesized $2 \mathrm{D} \mathrm{TIs}{ }^{9-23}$ in which square and hexagonal atomic rings are almost the only building blocks. We show, for the first time, that the QSH effect can also occur in the supposedly ordinary 2D crystals composed entirely of pentagonal rings. Our work opens new avenues for the discovery of additional large-gap 2D TIs in different and unexplored classes of systems.

To further reveal the nontrivial topological nature of these systems, we perform calculations to examine the topological edge states on the boundaries of $\alpha-\mathrm{SnX}_{2}, \beta-\mathrm{SnSe}_{2}$ and $\beta-\mathrm{SnTe}_{2} 2 \mathrm{D}$ crystals. They are calculated using a nanoribbon structure with symmetric edges, which leads to two energetically degenerate edge states located at opposite sides. The corresponding schematic ribbon model is shown in Figure 3a. All the dangling bonds of the edge atoms are saturated by hydrogen atoms. The widths of the nanoribbons are set large enough to avoid interactions between the two edges. Figures $3 \mathrm{~b}-\mathrm{f}$ presents the 
a

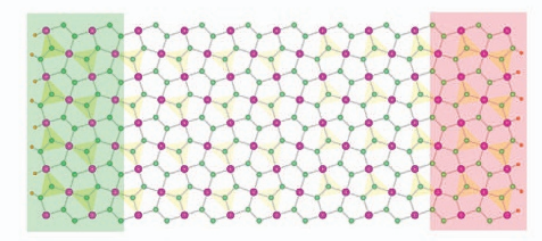

d

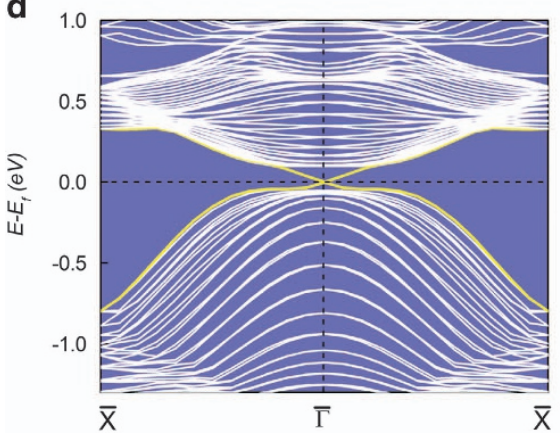

b

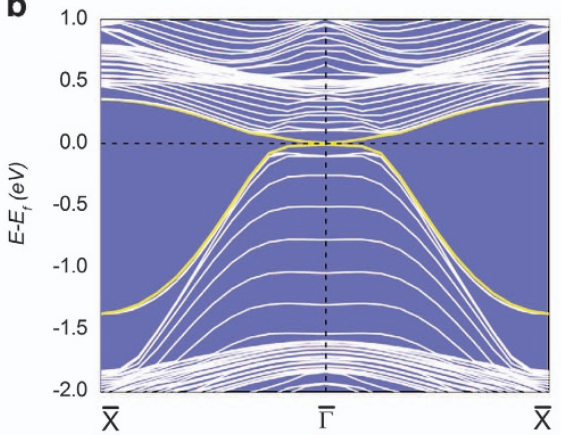

e

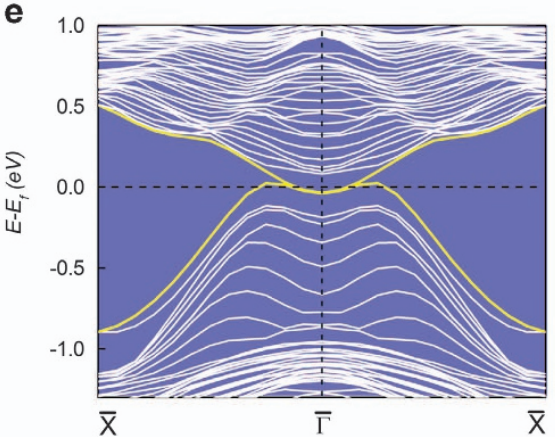

C

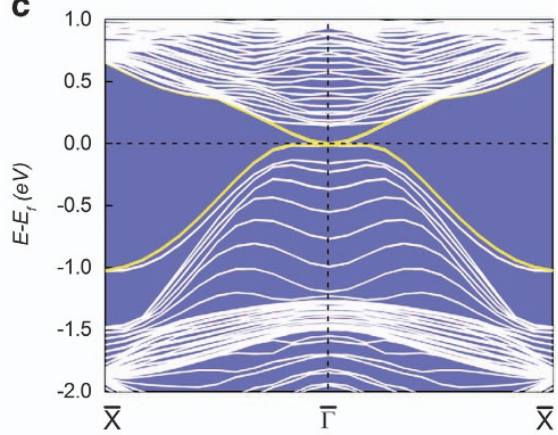

f

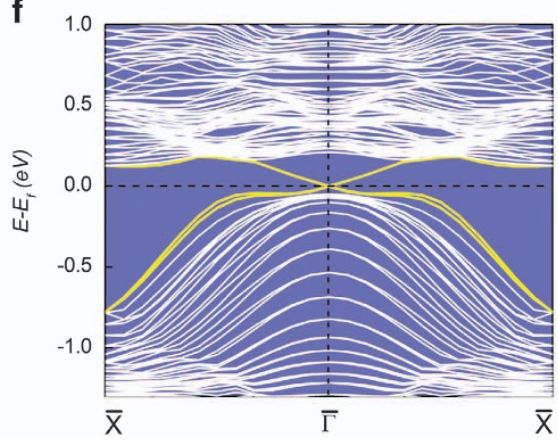

Figure 3 (a) Schematic $S n X_{2}$ ribbon model used to calculate the edge states. Pink and cyan balls denote $S n$ and $X$ atoms, respectively. Electronic band structures of (b) $\alpha-\mathrm{SnS}_{2}$, (c) $\alpha$-SnSe $\mathrm{Sn}_{2}$, (d) $\alpha-\mathrm{SnTe}_{2}$, (e) $\beta-\mathrm{SnSe}_{2}$ and (f) $\beta-\mathrm{SnTe}_{2}$ nanoribbons. The helical edge states dispersing in the bulk gap are visualized by the yellow lines. Fermi levels are set to zero.

calculated band structures of $\alpha-\mathrm{SnX}_{2}, \beta-\mathrm{SnSe}_{2}$ and $\beta$-SnTe $\mathrm{S}_{2}$ nanoribbons. One can explicitly see that each edge has a single pair of helical edge states (yellow lines) for all five of these systems, and the helical edge states disperse in the bulk band gap and cross linearly at the $\bar{\Gamma}$ point. These features further prove the nontrivial nature of these systems, which is consistent with the $Z_{2}$ calculations. Remarkably, in the cases of $\alpha-\mathrm{SnS}_{2}, \alpha-\mathrm{SnSe}_{2}, \alpha-\mathrm{SnTe}_{2}$ and $\beta-\mathrm{SnTe}_{2}$, the Dirac points formed by the helical edge states lie exactly at the Fermi level. Such placement of Dirac points is important for actual applications. Moreover, their sizeable bulk band gaps can stabilize the edge states against the interference of the thermally activated carriers, which are beneficial for observing the room-temperature QSH effect in these 2D crystals.

\section{DISCUSSION}

In the following section, we take $\alpha-\mathrm{SnTe}_{2} 2 \mathrm{D}$ crystal as an example and discuss the realistic possibility of experimentally realizing these $2 \mathrm{D}$ crystals. We find that the widely used square $\mathrm{BiOBiS}_{2}$ sheet ${ }^{39}$ can be used as a suitable substrate for an $\alpha-S_{n T e} 2 \mathrm{D}$ crystal in experiments. The in-plane lattice constant of an $\alpha-\mathrm{SnTe}_{2} 2 \mathrm{D}$ crystal is very close to that of the insulating $2 \times 2 \mathrm{BiOBiS}_{2}$ sheet. The lattice mismatch between them is only $2.3 \%$. To this end, we propose a quantum well structure between the $\alpha-\mathrm{SnTe}_{2} 2 \mathrm{D}$ crystal and $\mathrm{BiOBiS}_{2}$ sheet. As shown in Figure $4 \mathrm{a}$, in the $\mathrm{BiOBiS}_{2} / \alpha-\mathrm{SnTe}_{2}$ quantum well, one $\alpha-\mathrm{SnTe}_{2}$ layer is sandwiched between two $\mathrm{BiOBiS}_{2}$ sheets. Our calculated band structure displayed in Figure $4 \mathrm{~b}$ shows that the states around the Fermi level mainly come from the $p_{z}{ }^{-}$and $p_{x, y}{ }^{-}$orbitals of $\alpha-\mathrm{SnTe}_{2}$. In the absence of SOC for the $\mathrm{BiOBiS}_{2} / \alpha-\mathrm{SnTe}_{2}$ quantum well, which is similar to the case of a free-standing $\alpha-\mathrm{SnTe}_{2} 2 \mathrm{D}$ crystal, there is still one energy band with the $p_{x, y}{ }^{-}$orbital character that overlaps with another energy band with the $p_{x, y}{ }^{-}$orbital character around the Fermi level. However, such band overlapping only forms one Dirac point located at the Fermi level along the $\Gamma-X$ direction. After including SOC, the Dirac point is deformed, and a band gap appears, which is a strong indication for the existence of a topologically nontrivial phase. Therefore, the $\mathrm{BiOBiS}_{2} / \alpha-\mathrm{SnTe}_{2}$ quantum well may still be a 2D TI. To firmly confirm its band topology, we investigated the $Z_{2}$ topological invariant. The corresponding results are listed in Supplementary Table S3. The result of $Z_{2}=1$ indicates that a $\mathrm{BiOBiS}_{2} / \alpha-\mathrm{SnTe}_{2}$ quantum well is indeed a QSH insulator. More remarkably, the nontrivial bulk band gap is $44 \mathrm{meV}$, which is still well above the energy scale of room temperature.

To deeply understand the effect of the insulating substrate $\mathrm{BiOBiS}_{2}$ sheet on the topological nature of an $\alpha-\mathrm{SnTe}_{2} 2 \mathrm{D}$ crystal, we investigated the related properties of an isolated $\alpha-\mathrm{SnTe}_{2}$ layer that is taken directly from the $\mathrm{BiOBiS}_{2} / \alpha-\mathrm{SnTe}_{2}$ quantum well without any further structure relaxation. The corresponding band structures are plotted in Figure 4c, and we can observe that the band structure near the Fermi level retains linear band crossing in the absence of SOC, which resembles that of the $\mathrm{BiOBiS}_{2} / \alpha-\mathrm{SnTe}_{2}$ quantum well. When including SOC, a band gap of $214 \mathrm{meV}$ is produced in the isolated $\alpha-\mathrm{SnTe}_{2}$ layer. Our band topology analysis estimates that the topologically nontrivial state remains intact in the isolated $\alpha-\mathrm{SnTe}_{2}$ layer. However, by comparing Figures $4 \mathrm{~b}$ and $\mathrm{c}$, it is important to notice that the nontrivial band gap is significantly reduced (from 214 to $44 \mathrm{meV}$ ) when the isolated $\alpha-\mathrm{SnTe}_{2}$ layer is sandwiched between the $\mathrm{BiOBiS}_{2}$ sheets. This reduction reflects the band realignment effect when placing thin films on the substrate. The conduction band maximum and valence band maximum of the $\mathrm{BiOBiS}_{2} / \alpha-\mathrm{SnTe}_{2}$ quantum well are contributed by the $\mathrm{BiOBiS}_{2}$ sheets and isolated $\alpha$ $\mathrm{SnTe}_{2}$ layer, respectively. Obviously, these are the unique features of the type-II quantum well: the electron sub-band and hole sub-band are separated in two different layers. Considering these results, one can easily understand this reduction. Furthermore, these results also reveal 


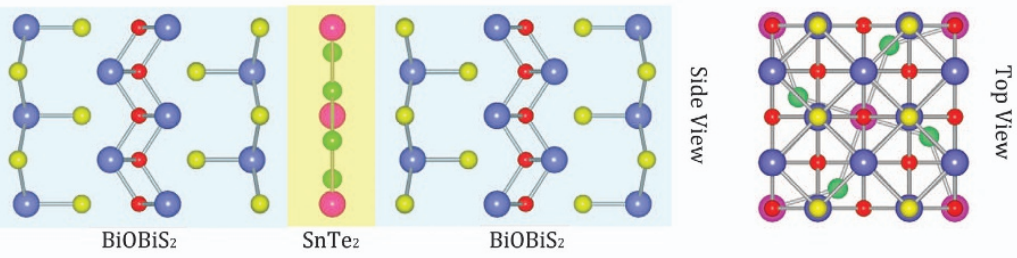

b
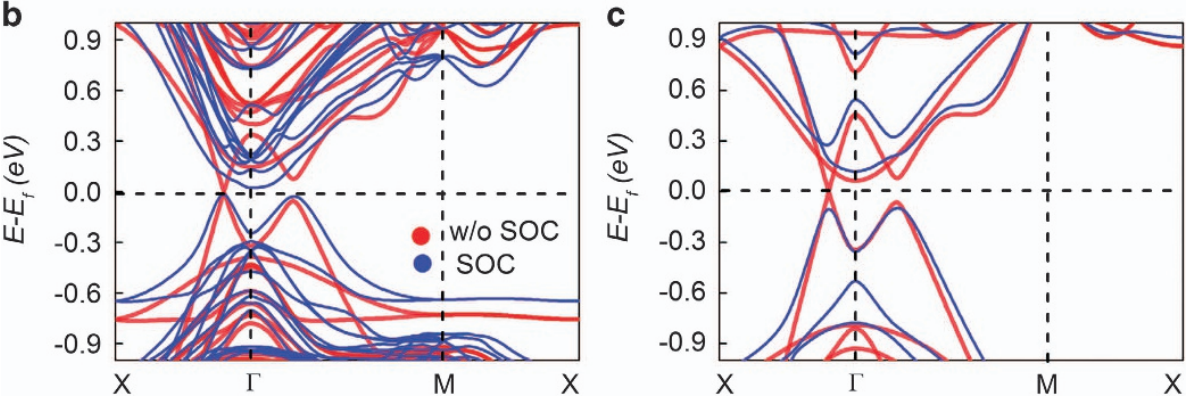

Figure 4 (a) Side and top views of the structural model for the BiOBiS $/ \alpha-\mathrm{SnTe}_{2}$ quantum well. Pink, cyan, blue, red and yellow balls denote $\mathrm{Sn}, \mathrm{Te}, \mathrm{Bi}, \mathrm{O}$ and $\mathrm{S}$ atoms, respectively. Electronic band structures of (b) the BiOBiS $2 / \alpha-\mathrm{SnTe}_{2}$ quantum well and (c) the isolated $\alpha$-SnTe $\mathrm{Stwo}_{2}$-dimensional (2D) crystal without and with spin-orbit coupling (SOC). The isolated $\alpha-\mathrm{SnTe}_{2} 2 \mathrm{D}$ crystal is taken from the $\mathrm{BiOBiS}_{2} / \alpha-\mathrm{SnTe}_{2}$ quantum well without any further structure relaxation. Fermi levels are set to zero.

the importance of the gap size of the substrate for maintaining the nontrivial topological states.

Finally, we wish to note that topological states have also been reported recently in IV-VI $2 \mathrm{D}$ crystals. ${ }^{40}$ Unlike the $\mathrm{SnX}_{2}$-based 2D TIs studied here, the topological states of IV-VI 2D crystals are protected by crystal symmetry instead of by time-reversal symmetry. Namely, these IV-VI 2D crystals are 2D topological crystalline insulators. ${ }^{40}$ Therefore, these two classes of $2 \mathrm{D}$ crystals with the same elements but different compositions/structures can lead to very distinct topological phases: $2 \mathrm{D}$ TI and 2D topological crystalline insulator. This finding is certainly interesting and suggests greater room in the design of topological materials beyond conventional structures.

In summary, we show by first-principles calculations that $\alpha$-SnX $\mathrm{X}_{2}$, $\beta-\mathrm{SnSe}_{2}$ and $\beta-\mathrm{SnTe}_{2} 2 \mathrm{D}$ crystals, which are composed exclusively of pentagonal rings, are $2 \mathrm{D}$ TIs. This result challenges the current knowledge 2D TIs, that is, that square or hexagonal rings are the commonly building blocks. Their topologically nontrivial characteristics are confirmed by the nontrivial $\mathrm{Z}_{2}$-type topological invariants and the existence of topologically protected helical edge states. Sizeable nontrivial band gaps of 121-224 meV are obtained, suggesting that the QSH effect can be realized in these systems above room temperature. For $\beta-\mathrm{SnSe}_{2}$ and $\beta-\mathrm{SnTe}_{2} 2 \mathrm{D}$ crystals, their bands exhibit the SOCinduced spin-splitting effect due to their broken inversion symmetry. $\alpha-\mathrm{SnX}_{2} 2 \mathrm{D}$ crystals are inversion symmetric; thus, no spin splitting can be observed. To probe the possibility of experimentally realizing these $2 \mathrm{D}$ crystals, we further propose the $\mathrm{BiOBiS}_{2} / \alpha-\mathrm{SnTe}_{2}$ quantum well. Additionally, we find that the $\alpha-\mathrm{SnTe}_{2} 2 \mathrm{D}$ crystal remains topologically nontrivial with a sizeable gap $(44 \mathrm{meV})$ when sandwiched between the $\mathrm{BiOBiS}_{2}$ sheets, thus suggesting that the 2D TIs predicted here can be sandwiched between appropriate nanostructures for realistic applications. Our work predicts that more pentagonal ring-based 2D TIs will be discovered in the future, which will greatly broaden the scientific and technological impact of the QSH effect.

\section{CONFLICT OF INTEREST}

The authors declare no conflict of interest.

\section{ACKNOWLEDGEMENTS}

Financial support from the European Research Council (ERC, StG 256962) and the Taishan Scholar Program of Shandong is gratefully acknowledged.

Author contributions: YM and TH conceived and designed this study, YM performed the calculations, and all authors discussed the results and contributed to the writing of the manuscript.

1 Hasan, M. Z. \& Kane, C. L. Colloquium: topological insulators. Rev. Mod. Phys. 82, 3045-3067 (2010)

2 Qi, X.-L. \& Zhang, S.-C. Topological insulators and superconductors. Rev. Mod. Phys. 83, 1057 (2011).

3 Kane, C. L. \& Mele, E. J. Quantum spin Hall effect in graphene. Phys. Rev. Lett. 95, 226801 (2005); Phys. Rev. Lett. $\mathrm{Z}_{2}$ topological order and the quantum spin Hall effect. 95,146802 (2005).

4 Bernevig, B. A. \& Zhang, S. C. Quantum spin Hall effect. Phys. Rev. Lett. 96, 106802 (2006).

5 Zhang, H., Liu, C.-X., Qi, X.-L., Dai, X., Fang, Z. \& Zhang, S.-C. Topological insulators in $\mathrm{Bi}_{2} \mathrm{Se}_{3}, \mathrm{Bi}_{2} \mathrm{Te}_{3}$ and $\mathrm{Sb}_{2} \mathrm{Te}_{3}$ with a single Dirac cone on the surface. Nat. Phys. 5, 438-442 (2009).

6 Xia, Y., Qian, D., Hsieh, D., Wray, L., Pal, A., Lin, H., Bansil, A., Grauer, D., Hor, Y. S., Cava, R. J. \& Hasan, M. Z. Observation of a large-gap topological-insulator class with a single Dirac cone on the surface. Nat. Phys. 5, 398-402 (2009).

7 Konig, M., Wiedmann, S., Brune, C., Roth, A., Buhmann, H., Molenkamp, L. W., Qi, X. L. \& Zhang, S. C. Quantum spin Hall insulator state in HgTe quantum wells. Science 318, 766-770 (2007).

8 Knez, I., Du, R.-R. \& Sullivan, G. Evidence for helical edge modes in inverted InAs/ GaSb quantum wells. Phys. Rev. Lett. 107, 136603 (2011).

9 Liu, C.-C., Feng, W. \& Yao, Y. Quantum spin Hall effect in silicene and two-dimensional germanium. Phys. Rev. Lett. 107, 076802 (2011).

10 Murakami, S. Quantum spin Hall effect and enhanced magnetic response by spin-orbit coupling. Phys. Rev. Lett. 97, 236805 (2006).

11 Chuang, F.-C., Yao, L.-Z., Huang, Z.-Q., Liu, Y.-T., Hsu, C.-H., Das, T., Lin, H. \& Bansil, A. Prediction of large-gap two-dimensional topological insulators consisting of bilayers of group III elements with Bi. Nano Lett 14, 2505 (2014).

12 Luo, W. \& Xiang, H. J. Room temperature quantum spin Hall insulators with a buckled square lattice. Nano Lett. 15, 3230-3235 (2015).

13 Zhou, J. J., Feng, W. X., Liu, C.-C., Guan, S. \& Yao, Y. G. Large-gap quantum spin Hall insulator in single layer bismuth monobromide $\mathrm{Bi}_{4} \mathrm{Br}_{4}$. Nano Lett. 14, 4767-4771 (2014) 
14 Ma, Y. D., Kou, L. Z., Du, A. J. \& Heine, T. Group 14 element-based noncentrosymmetric quantum spin Hall insulators with large bulk gap. Nano Res. 8 3412-3420 (2015).

15 Si, C., Liu, J. W., Xu, Y., Wu, J., Gu, B.-L. \& Duan, W. H. Functionalized germanene as a prototype of large-gap two-dimensional topological insulators. Phys. Rev. B 89, 115429 (2014).

16 Xu, Y., Yan, B. H., Zhang, H.-J., Wang, J., Xu, G., Tang, P., Duan, W. \& Zhang, S. C. Large-gap quantum spin Hall insulators in tin films. Phys. Rev. Lett. 111, 136804 (2013).

$17 \mathrm{Ma}$, Y. D., Dai, Y., Kou, L. Z., Frauenheim, T. \& Heine, T. Robust two-dimensional topological insulators in methyl-functionalized bismuth, antimony, and lead bilayer films. Nano Lett. 15, 1083-1089 (2015).

18 Song, Z. G., Liu, C. C., Yang, J. B., Han, J. Z., Fu, B. T., Ye, M., Fu, B. T., Yang, Y. C. Niu, Q., Lu, J., Yao, Y. G. Quantum spin Hall insulators and quantum valley Hall insulators of $\mathrm{BiX} / \mathrm{SbX}(\mathrm{X}=\mathrm{H}, \mathrm{F}, \mathrm{Cl}$ and $\mathrm{Br})$ monolayers with a record bulk band gap. NPG Asia Mater. 6, 147 (2014).

19 Weng, H., Dai, X. \& Fang, Z. Transition-metal pentatelluride $\mathrm{ZrTe}_{5}$ and $\mathrm{HfTe}_{5}$ : a paradigm for large-gap quantum spin Hall insulators. Phys. Rev. X 4, 011002 (2014).

20 Ma, Y. D., Kou, L. Z., Li, X., Dai, Y., Smith, S. C. \& Heine, T. Quantum spin Hall effect and topological phase transition in two-dimensional square transition-metal dichalcogenides. Phys. Rev. B 92, 085427 (2015).

$21 \mathrm{Ma}$, Y. D., Kou, L. Z., Li, X., Dai, Y. \& Heine, T. Two-dimensional transition metal dichalcogenides with a hexagonal lattice: room-temperature quantum spin Hall insulators. Phys. Rev. B 93, 035442 (2016).

22 Nie, S. M., Song, Z., Weng, H. M. \& Fang, Z. Quantum spin Hall effect in twodimensional transition-metal dichalcogenide haeckelites. Phys. Rev. B 91, 235434 (2015).

23 Qian, X. F., Liu, J. W., Fu, L. \& Li, J. Quantum spin Hall effect in two-dimensional transition metal dichalcogenides. Science 346, 1344-1347 (2014).

24 Zhang, S. H., Zhou, J., Wang, Q., Chen, X. S., Kawazoe, Y. \& Jena, P. Penta-graphene: a new carbon allotrope. Proc Natl Acad Sci USA 112, 2372-2377 (2015).

25 Tang, C. P., Xiong, S. J., Shi, W. J. \& Cao, J. Two-dimensional pentagonal crystals and possible spin-polarized Dirac dispersion relations. J. Appl. Phys. 115, 113702 (2014)

26 Kresse, G. \& Furthmüller, J. Efficiency of ab-initio total energy calculations for metals and semiconductors using a plane-wave basis set. Comput. Mater. Sci. 6, 15-50 (1996)

27 Kresse, G. \& Furthmller, J. Efficient iterative schemes for ab initio total-energy calculations using a plane-wave basis set. Phys. Rev. B 54, 11169 (1996).

28 Perdew, J. P., Burke, K. \& Ernzerhof, M. Generalized gradient approximation made simple. Phys. Rev. Lett. 77, 3865 (1996).

29 Perdew, J. P. \& Wang, Y. Accurate and simple analytic representation of the electrongas correlation energy. Phys. Rev. B 45, 13244 (1992).
30 Monkhorst, H. J. \& Pack, J. D. Special points for Brillouin-zone integrations. Phys. Rev. B 13, 5188 (1976).

31 Grimme, S. Semiempirical GGA-type density functional constructed with a long-range dispersion correction. J. Comput. Chem. 27, 1787-1799 (2006).

32 Schmidt, C. L., Dinnebier, R., Wedig, U. \& Jansen, M. Crystal structure and chemica bonding of the high-temperature phase of $\mathrm{AgN}_{3}$. Inorg. Chem. 46, 907-916 (2007).

33 Ressouche, E., Simonet, V., Canals, B., Gospodinov, M. \& Skumryev, V. Magnetic frustration in an iron-based Cairo pentagonal lattice. Phys. Rev. Lett. 103, 267204 (2009).

34 Zhou, L. J., Kou, L. Z., Sun., Y., Felser, C., Hu, F. M., Shan, G. C., Smith, S. C., Yan, B. \& Frauenheim, T. New family of quantum spin Hall insulators in two-dimensional transition-metal halide with large nontrivial band gaps. Nano Lett. 15 7867-7872 (2015)

35 Huang, H. Q., Liu, J. P. \& Duan, W. H. Nontrivial $Z_{2}$ topology in bismuth-based III-V compounds. Phys. Rev. B 90, 195105 (2014).

36 Zhang, Y., He, K., Chang, C.-Z., Song, C.-L., Wang, L.-L., Chen, X., Jia, J.-F., Fang, Z., Dai, X., Shan, W.-Y., Shen, S.-Q., Niu, Q., Qi, X.-L., Zhang, S. C., Ma, X.-C. \& Xue, Q.-K. Crossover of the three-dimensional topological insulator $\mathrm{Bi}_{2} \mathrm{Se}_{3}$ to the twodimensional limit. Nat. Phys. 6, 584-588 (2010).

$37 \mathrm{Fu}$, L., Kane, C. L. \& Mele, E. J. Topological insulators in three dimensions. Phys. Rev. Lett. 98, 106803 (2007).

38 Fukui, T. \& Hatsugai, Y. Quantum spin Hall effect in three dimensional materials: Lattice computation of $\mathrm{Z}_{2}$ topological invariants and its application to $\mathrm{Bi}$ and $\mathrm{Sb}$. J. Phys. Soc. Jpn. 76, 053702 (2007)

39 Phelan, W. A., Wallace, D. C., Arpino, K. E., Neilson, J. R., Livi, K. J., Seabourne, C. R., Scott, A. J. \& McQueen, T. M. Stacking variants and superconductivity in the Bi-0-S system. J. Am. Chem. Soc. 135, 5372-5374 (2013).

40 Liu, J. W., Qian, X. F. \& Fu, L. Crystal field effect induced topological crystalline insulators in monolayer IV - VI semiconductors. Nano Lett. 15, 2657-2661 (2015).

(i) This work is licensed under a Creative Commons Attribution 4.0 International License. The images or other third party material in this article are included in the article's Creative Commons license, unless indicated otherwise in the credit line; if the material is not included under the Creative Commons license, users will need to obtain permission from the license holder to reproduce the material. To view a copy of this license, visit http:// creativecommons.org/licenses/by/4.0/

Supplementary Information accompanies the paper on the NPG Asia Materials website (http://www.nature.com/am) 